\title{
The Explicit Solution of Model Predictive Control via Multiparametric Quadratic Programming
}

\author{
Alberto Bemporad ${ }^{\dagger}$, Manfred Morari $^{\dagger}$, Vivek Dua ${ }^{\ddagger}$, Efstratios N. Pistikopoulos ${ }^{\ddagger}$ \\ ${ }^{\dagger}$ Automatic Control Laboratory \\ ETH Zentrum, ETL I 26 \\ CH-8092 Zurich, Switzerland \\ Tel. +41-1-632 6679, Fax +41-1-632 1211 \\ bemporad, moraridaut.ee.ethz.ch \\ ‡Centre for Process Systems Engineering \\ Imperial College \\ London SW7 2BY, U.K. \\ Tel +44-171-594 6620, Fax +44-171-5946606 \\ v.dua, e.pistikopoulos@ic.ac.uk
}

\begin{abstract}
Control based on on-line optimization, popularly known as model predictive control (MPC), has long been recognized as the winning alternative for constrained systems. The main limitation of MPC is, however, its on-line computational complexity. For discrete-time linear time-invariant systems with constraints on inputs and states, we develop an algorithm to determine explicitly the state feedback control law associated with MPC, and show that it is piecewise linear and continuous. The controller inherits all the stability and performance properties of MPC, but the online computation is reduced to a simple linear function evaluation instead of the expensive quadratic program. The new technique is expected to enlarge the scope of applicability of MPC to small-size/fast-sampling applications which cannot be covered satisfactorily with anti-windup schemes.
\end{abstract}

\section{Introduction}

As we extend the class of system descriptions beyond the class of linear systems, linear systems with constraints are probably the most important class in practice. The most popular approaches for designing controllers for linear systems with constraints fall into two categories: anti-windup and model predictive control. Anti-windup schemes assume that a well functioning linear controller is available for small excursions from the nominal operating point. This controller is augmented by the anti-windup scheme in a somewhat ad hoc fashion to take care of situations when constraints are met. Kothare et al. [13] reviewed numerous apparently different anti-windup schemes and showed that they differ only in their choice of two static matrix parameters. Anti-windup schemes are widely used in practice because in most SISO situations they are simple to design and work adequately.

Model Predictive Control (MPC) has become the accepted standard for complex constrained multivariable control problems in the process industries. Here at each sampling time, starting at the current state, an open-loop optimal control problem is solved over a finite horizon. At the next time step the computation is repeated starting from the new state and over a shifted horizon, leading to a moving horizon policy. The solution relies on a linear dynamic model, respects all input and output constraints, and optimizes a quadratic performance index. The big drawback of MPC is the relatively formidable on-line computational effort which limits its applicability to relatively slow and/or small problems.

In this paper we show how to move all the computations necessary for the implementation of MPC offline while preserving all its other characteristics. This should largely increase MPC's range of applicability to problems where anti-windup schemes and other ad hoc techniques dominated up to now.

The paper is organized as follows. The basics of MPC are reviewed first to derive the quadratic program which needs to be solved to determine the optimal control action. We note that the quadratic program depends on the current state which appears linearly in the constraints, i.e., it is a multi-parametric quadratic program. Next we study the multi-parametric quadratic programming problem. We show that the optimal solution is a piecewise affine function of the state (confirming previous investigations on the form of MPC laws $[16,17,7])$, analyze its properties, and develop an efficient algorithm to solve it. The paper concludes with an example which illustrates the different features of the method.

The results in this paper can be extended easily to 1norm and $\infty$-norm objective functions instead of the 2 -norm employed in this paper. The resulting multiparametric linear program can be solved in a similar manner as suggested here [6], or as in [10]. For MPC of hybrid systems, an extension involving multiparametric mixed-integer linear programming, is also possible [3].

\section{Model Predictive Control}

Consider the problem of regulating to the origin the discrete-time linear time invariant system

$$
\left\{\begin{aligned}
x(t+1) & =A x(t)+B u(t) \\
y(t) & =C x(t)
\end{aligned}\right.
$$

while fulfilling the constraints

$$
y_{\min } \leq y(t) \leq y_{\max }, u_{\min } \leq u(t) \leq u_{\max }
$$

at all time instants $t \geq 0$. In (1)-(2), $x(t) \in \mathbb{R}^{n}$, $u(t) \in \mathbb{R}^{m}$, and $y(t) \in \mathbb{R}^{p}$ are the state, input, and output vector respectively, $y_{\min } \leq y_{\max }\left(u_{\min } \leq u_{\max }\right)$ 
are bounds on outputs (inputs), and the pair $(A, B)$ is stabilizable.

Model Predictive Control (MPC) solves such a constrained regulation problem in the following way. Assume that a full measurement of the state $x(t)$ is available at the current time $t$. Then, the optimization problem

$$
\begin{array}{ll}
\underset{U \triangleq\left\{u_{t}, \ldots, u_{t+N_{u}-1}\right\}}{\operatorname{problem}} & \left\{J(U, x(t))=x_{t+N_{y} \mid t}^{\prime} P x_{t+N_{y} \mid t}\right. \\
& \left.+\sum_{k=0}^{N_{y}-1} x_{t+k \mid t}^{\prime} Q x_{t+k \mid t}+u_{t+k}^{\prime} R u_{t+k}\right\}
\end{array}
$$

subj. to

$$
\begin{aligned}
& y_{\min } \leq y_{t+k \mid t} \leq y_{\max }, k=1, \ldots, N_{c} \\
& u_{\min } \leq u_{t+k} \leq u_{\max }, k=0,1, \ldots, N_{c} \\
& x_{t \mid t}=x(t) \\
& x_{t+k+1 \mid t}=A x_{t+k \mid t}+B u_{t+k}, k \geq 0 \\
& u_{t+k}=K x_{t+k \mid t}, N_{u} \leq k<N_{y}
\end{aligned}
$$

is solved at each time $t$, where $x_{t+k \mid t}$ denotes the predicted state vector at time $t+k$, obtained by applying the input sequence $u_{t}, \ldots, u_{t+k-1}$ to model (1) starting from the state $x(t)$. In (3), we assume that $Q=Q^{\prime} \succeq 0$, $R=R^{\prime} \succ 0, P \succeq 0,\left(Q^{\frac{1}{2}}, A\right)$ detectable (for instance $Q=C^{\prime} C$ with $(\bar{C}, A)$ detectable $), N_{y} \geq N_{u}$, and $K$ is some feedback gain.

One possibility is to choose $K=0$ [14], and $P$ as the solution of the Lyapunov equation $P=A^{\prime} P A+Q$. This is only meaningful when the system is open-loop stable, as $J(U, x(t))$ measures the settling cost of the system from the present time $t$ to infinity under the assumption that the control is turned off after $N_{u}$ steps.

Alternatively, one can set $K=K_{L Q}$ and $P$ as the solution of the unconstrained infinite horizon LQR problem with weights $Q, R$. This choice of $K$ implies that after $N_{u}$ time steps the control is switched to the unconstrained LQR.

Let $U^{*}(t)=\left\{u_{t}^{*}, \ldots, u_{t+N_{u}-1}^{*}\right\}$ be the optimal solution of $(3)$. Then at time $t$

$$
u(t)=u_{t}^{*}
$$

is applied as input to system (1).

Most approaches for proving stability follow in spirit the arguments of Keerthi and Gilbert [12] who establish the fact that under some conditions the value function $V(t)=J\left(U^{*}(t), t\right)$ attained at the minimizer $U^{*}(t)$ is a Lyapunov function of the system. Below we recall a simple stability result based on such a Lyapunov argument, whose proof is reported in[5].

Theorem 1 Let $N_{y}=\infty, K=0$ or $K=K_{L Q}$, and $N_{c}<\infty$ be computed according to the theory of the maximal output admissible set [11, 2]. Then the MPC law (3)-(4) is asymptotically stabilizing for system (1) while fulfilling the constraints (2) from all initial states $x(0)$ such that (3) is feasible at $t=0$.

By substituting $x_{t+k \mid t}=A^{k} x(t)+\sum_{j=0}^{k-1} A^{j} B u_{t+k-1-j}$ in (3), the optimization problem (3) can be rewritten in the form

$$
\begin{aligned}
V(x(t))=\frac{1}{2} x^{\prime}(t) Y x(t)+ & \min _{U} \frac{1}{2} U^{\prime} H U+x^{\prime}(t) F U \\
& \text { subj. to } G U \leq W+E x(t)
\end{aligned}
$$

where the column vector $U \triangleq\left[u_{t}^{\prime}, \ldots, u_{t+N_{u}-1}^{\prime}\right]^{\prime} \in \mathbb{R}^{s}$, $s \triangleq m N_{u}$, is the optimization vector, $H=H^{\prime} \succ 0$, and $H, F, Y, G, W, E$ are easily obtained from $Q, R$, and (3) (as only the optimizer $U$ is needed, the term involving $Y$ is usually removed from (5)).

The optimization problem (5) is a quadratic program (QP). Because the problem depends on the current state $x(t)$, implementation of MPC requires the online solution of a QP at each time step. Although efficient QP solvers based on active-set methods or interior point methods are available, computing the input $u(t)$ demands significant on-line computation effort. For this reason, the application of MPC has been limited to "slow" and/or "small" processes.

In this paper we propose a new approach to implement MPC, where all the computation effort is moved offline. The idea is based on the observation that in (5), the state $x(t) \in \mathbb{R}^{n}$ can be considered a vector of parameters. In other words, the state feedback control law is defined implicitly as the solution of the optimization problem (5) as a function of the parameter $x(t)$. Our goal is to make this dependence explicit.

The operations research community has addressed problems depending on a vector of parameters as multi-parametric programs. According to this terminology, (5) is a multi-parametric Quadratic Program (mp-QP). Most of the literature deals with parametric problems, but some authors have addressed the multiparametric case $[9,10,8]$.To the authors' knowledge, no algorithm for solving mp-QP problems has been published. Once the multi-parametric problem (5) has been solved off line, i.e. the solution $U_{t}^{*}=f(x(t))$ of (5) has been found, the model predictive controller (3) is available explicitly, as the optimal input $u(t)$ consists simply of the first $m$ components of $U_{t}^{*}, u(t)=$ $\left[\begin{array}{llll}I & 0 & \ldots & 0\end{array}\right] f(x(t))$. In Section 3, we will show that the solution $U^{*}=f(x)$ of the mp-QP problem is continuous and piecewise affine (the same properties are inherited by the controller) and describe an algorithm to solve $\mathrm{mp}-\mathrm{QP}$ problems.

\subsection{Extensions}

Extensions of the basic MPC algorithm which can be expressed as an mp-QP (resulting in a piecewise affine controller) include: reference tracking, where the explicit MPC law is $\delta u(t)=F(x(t), u(t-1), r(t))$, with $\delta u(t)=u(t)-u(t-1)$ and $r(t)$ represents the desired reference; rejection of measured disturbances $v(t)$, where the resulting law is a piecewise affine function $u(t)=F(x(t), v(t)) ;$ soft constraints on outputs, where a slack variable $\epsilon$ is introduced into the constraints to relax the " $\leq$ " condition and added to the objective to penalize constraint violations ( $\epsilon$ plays the role of an independent optimization variable in the mp-QP. The form of the solution $u(t)=F(x(t))$ is not affected by this modification); variable constraints, where the bounds $y_{\min }, y_{\max }, \delta u_{\min }, \delta u_{\max }, u_{\min }, u_{\max }$ are treated as parameters, and may change depending on the operating conditions (in the case of a stuck actuator the constraints become $\delta u_{\min }=\delta u_{\max }=0$ ).

2.2 Piecewise Linear Solution of the Constrained Linear Quadratic Regulation Problem In their pioneering work [15], Sznaier and Damborg 
showed that finite horizon optimization (3), (4), with $P$ satisfying the algebraic Riccati equation also provides the solution to the infinite-horizon linear quadratic regulation problem with constraints (C-LQR). This idea has been reconsidered later by other authors. The equivalence holds for a certain set of initial conditions, which depends on the length of the finite horizon. The multiparametric programming approach proposed here has been considered in [5] to provide the explicit solution to infinite horizon C-LQR problems.

\section{Multi-Parametric Quadratic Programming}

In this section we investigate multi-parametric quadratic programs (mp-QP) of the form (5). Before proceeding further, it is useful to define

$$
z \triangleq U+H^{-1} F^{\prime} x(t),
$$

$z \in \mathbb{R}^{s}$, and to transform (5) by completing squares to obtain the equivalent problem

$$
\begin{aligned}
V_{z}(x)= & \min _{z} \frac{1}{2} z^{\prime} H z \\
& \text { subj. to } \quad G z \leq W+S x(t),
\end{aligned}
$$

where $S \triangleq E+G H^{-1} F^{\prime}$, and $V_{z}(x)=V(x)-\frac{1}{2} x^{\prime}(Y-$ $\left.F H^{-1} F^{\prime}\right) x$. In the transformed problem the parameter vector $x$ appears only on the rhs of the constraints. The solution of mp-QP problems can be approached by employing the principles of parametric nonlinear programming, and in particular the first-order KarushKuhn-Tucker (KKT) optimality conditions [1]. In this paper, we adopt a more direct approach than [9], by exploiting the linearity of the constraints and the fact that the function to be minimized is quadratic. In order to use the KKT conditions (8), we assume the following condition to hold on the constraints in (7).

Assumption 1 For each $x \in X$, and for each admissible combination of active constraints in (7) at the minimizer, the corresponding rows of matrix $G$ are linearly independent.

Theorem 2 Let $H \succ 0$ and $G$ satisfy Assumption 1. Then for each feasible combination of active constraints, the optimal $z$ and Lagrange multipliers $\lambda$ are uniquely defined affine functions of $x$.

Proof: The first-order KKT conditions for the mpQP are given by

$$
\begin{aligned}
& H z+G^{\prime} \lambda=0, \lambda \in \mathbb{R}^{q} \\
& \lambda_{i}\left(G^{i} z-W^{i}-S^{i} x\right)=0, i=1, \ldots, q \\
& \lambda \geq 0
\end{aligned}
$$

where the superscript ${ }^{i}$ denotes the $i$-th row. We solve (8a) for $z$,

$$
z=-H^{-1} G^{\prime} \lambda
$$

and substitute the result into (8b) to obtain the complementary slackness condition $\lambda\left(-G H^{-1} G^{\prime} \lambda-W\right.$ $-S x)=0$. Let $\breve{\lambda}$ and $\tilde{\lambda}$ denote the Lagrange multipliers corresponding to inactive and active constraints, respectively. For inactive constraints, $\breve{\lambda}=0$. For active constraints, $-\tilde{G} H^{-1} \tilde{G}^{\prime} \tilde{\lambda}-\tilde{W}-\tilde{S} x=0$, and therefore

$$
\tilde{\lambda}=-\left(\tilde{G} H^{-1} \tilde{G}^{\prime}\right)^{-1}(\tilde{W}+\tilde{S} x)
$$

where $\tilde{G}, \tilde{W}, \tilde{S}$ correspond to the set of active constraints, and $\left(\tilde{G} H^{-1} \tilde{G}^{\prime}\right)^{-1}$ exists because of Assumption 1. Thus $\lambda$ is an affine function of $x^{1}$. We can substitute $\tilde{\lambda}$ from (10) into (9) to obtain

$$
z=H^{-1} \tilde{G}^{\prime}\left(\tilde{G} H^{-1} \tilde{G}^{\prime}\right)^{-1}(\tilde{W}+\tilde{S} x)
$$

and note that $z$ is also an affine function of $x$. A similar result was obtained by Zafiriou $[17,7]$.

However, his result does not make the piecewise linear dependence of $u$ on $x$ explicit, as the domains over which the different linear laws are defined are not characterized.

Theorem 2 characterizes the solution only locally in the neighborhood of a specific $x$. This characterization remains valid as long as the set of active constraints does not change as we change $x$. Choose an arbitrary vector of parameter values $x_{0} \in X$ and let $\left(z_{0}, \lambda_{0}\right)$ be the corresponding values satisfying the KKT triple (8), which are obtained by solving a QP for $x=x_{0}$. Assume that $G$ satisfies Assumption 1. Then, in a neighborhood of $x_{0}$, Theorem 2 allows to compute the linear function $[z(x), \lambda(x)]$, by simply looking at the constraints in (7) which are active at the minimizer $z_{0}$, and then building matrices $\tilde{G}, \tilde{W}$, and $\tilde{S}$ accordingly. The set of parameters $x$ where this combination of active constraints remains optimal is defined as the critical region $C R_{0}$. This region can be characterized easily. The variable $z$ from (9) must satisfy the constraints in (7)

$$
G H^{-1} \tilde{G}^{\prime}\left(\tilde{G} H^{-1} \tilde{G}^{\prime}\right)^{-1}(\tilde{W}+\tilde{S} x) \leq W+S x(t)
$$

and by (8c) the Lagrange multipliers in (10) must remain nonnegative

$$
-\left(\tilde{G} H^{-1} \tilde{G}^{\prime}\right)^{-1}(\tilde{W}+\tilde{S} x) \geq 0
$$

as we vary $x$. After removing the redundant inequalities from (12) and (13) we obtain a compact representation of $C R_{0}$. Obviously, $C R_{0}$ is a polyhedron in the $x$ space, and represents the largest set of $x \in X$ such that the combination of active constraints at the minimizer remains unchanged. Once the critical region $C R_{0}$ has been defined, the rest of the space $C R^{\text {rest }}=X-C R_{0}$ has to be explored and new critical regions generated. A possible approach for partitioning the rest of the space was proposed in [8], and is proven by the following theorem [5]

Theorem 3 Let $Y \subseteq \mathbb{R}^{n}$ a polyhedron, and $C R_{0} \triangleq$ $\{x \in Y: A x \leq b\}$ a polyhedral subset of $Y, C R_{0} \neq \emptyset$. Also let

$$
C R_{i}=\left\{x \in Y: \begin{array}{l}
A^{i} x>b^{i} \\
A^{j} x \leq b^{j}, \forall j<i
\end{array}\right\} i=1, \ldots, m
$$

where $m=\operatorname{dim}(b)$, and let $C R^{\text {rest }} \triangleq \cup_{i=1}^{m} C R_{i}$. Then

\footnotetext{
${ }^{1}$ As $\tilde{G} H^{-1} \tilde{G}^{\prime} \breve{\lambda}=\tilde{W}+\tilde{S} x$, the linear relation between $\lambda$ and $x$ still holds even if Assumption 1 is not satisfied.
} 
(i) $C R^{\text {rest }} \cup C R_{0}=Y$

(ii) $C R_{0} \cap C R_{i}=\emptyset, C R_{i} \cap C R_{j}=\emptyset, \forall i \neq j$

i.e. $\left\{C R_{0}, C R_{1}, \ldots, C R_{m}\right\}$ is a partition of $Y$.

The argument (10)-(13) is repeated in each new region $C R_{i}$, until the whole $x$-space has been covered. Then, those polyhedral regions $C R^{i}$ are determined where the function $z(x)$ is the same. If their union is a convex set, it is computed to permit a more compact description of the solution [4].

\subsection{Continuity and Convexity Properties}

Continuity of the value function $V_{z}(x)$ and the solution $z(x)$, can be shown as simple corollaries of the linearity result of Theorem 2. This fact, together with the convexity of the set of feasible parameters $X_{f} \subseteq X$ (i.e. the set of parameters $x \in X$ such that a feasible solution $z(x)$ exists to the optimization problem (7)), and of the value function $V_{z}(x), V(x)$, is proved in the next results. The proof is given in [5].

Theorem 4 Consider the multi-parametric quadratic program (7) and let $H \succ 0, X$ convex. Then the set of feasible parameters $X_{f} \subseteq X$ is convex, the optimizer $z(x): X_{f} \mapsto \mathbb{R}^{s}$ is continuous and piecewise affine, and the optimal solution $V_{z}(x): X_{f} \mapsto \mathbb{R}$ is continuous, convex and piecewise quadratic.

Lemma 1 Let $J(U, x)=\frac{1}{2} U^{\prime} H U+x^{\prime} F U+\frac{1}{2} x^{\prime} Y x$, and let $\left[\begin{array}{ll}Y & F^{\prime}\end{array}\right] \geq 0$. Then $V(x) \triangleq \min _{U} J(x, U)$ subject to $A U \stackrel{B}{\leq}+C x$ is a convex function of $x$.

Corollary 1 The value function $V(x)$ defined by the optimization problem (3), (5) is continuous and piecewise quadratic.

A simple consequence of Corollary 1 is that the Lyapunov function used to prove Theorem 1 is continuous, convex, and piecewise quadratic.

Corollary 2 The control law $u(t)=f(x(t)), f: \mathbb{R}^{n} \mapsto$ $\mathbb{R}^{m}$, defined by the optimization problem (3) and (4) is continuous and piecewise affine.

\subsection{Complexity of the Multiparametric QP Al- gorithm}

We addressed a solver for mp-QP which partitions the set of states $X$ in $N_{r}$ convex polyhedral regions. This number $N_{r}$ depends on the dimension $n$ of the state, the product $s=m N_{u}$ of the number $N_{u}$ of control moves and the dimension $m$ of the input vector, and the number of constraints $q$ in the optimization problem (7). Let $q_{s} \triangleq \operatorname{rank} S, q_{s} \leq q$. For $n>q_{s}$ the number of polyhedral regions $N_{r}$ remains constant. To see this, consider the linear transformation $\bar{x}=S x$, $\bar{x} \in \mathbb{R}^{q}$. Clearly $\bar{x}$ and $x$ define the same set of active constraints, and therefore the number of partitions in the $\bar{x}$ - and $x$-space are the same. Therefore, the number of partitions $N_{r}$ of the $x$-space defining the optimal controller is insensitive to the dimension $n$ of the state $x$ for all $n \geq q_{s}$, i.e. to the number of parameters involved in the mp-QP.
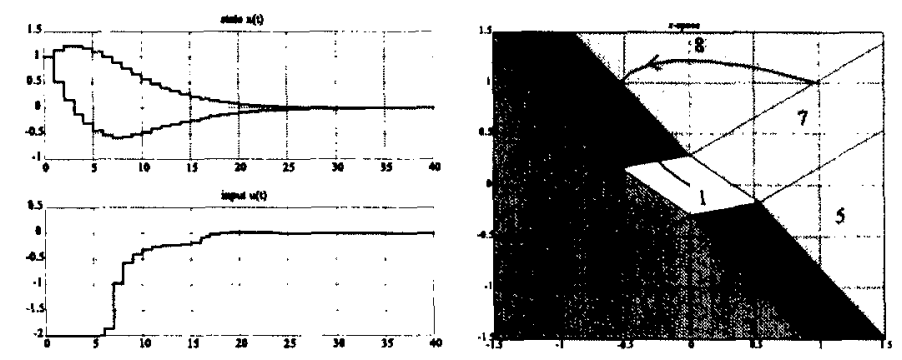

Figure 1: Closed-loop MPC and partition of the statespace

The number $q$ of constraints increases with $N_{c}$ and, in case of input constraints, with $N_{u}$. For instance, $q=$ $2 s=2 m N_{u}$ for control problems with input constraints only. From the analysis above, the larger $N_{c}, N_{u}, m, p$ the larger $q$, and therefore $N_{r}$. Note that many control problems involve input constraints only, and typically horizons $N_{u}=2$ or 3 and blocking of control moves are adopted, which reduces the number of constraints $q$.

\section{An Example}

Consider the second order system

$$
y(t)=\frac{2}{s^{2}+3 s+2} u(t)
$$

sample the dynamics with $T=0.1 \mathrm{~s}$, and obtain the state-space representation

$\left\{\begin{aligned} x(t+1) & =\left[\begin{array}{cc}0.7326 & -0.0861 \\ 0.1722 & 0.9909\end{array}\right] x(t)+\left[\begin{array}{l}0.0609 \\ 0.0064\end{array}\right] u(t) \\ y(t) & =\left[\begin{array}{ll}0 & 1.4142\end{array}\right] x(t)\end{aligned}\right.$

The task is to regulate the system to the origin while fulfilling the input constraint $-2 \leq u(t) \leq 2$. To this aim, we design an MPC controller based on the optimization problem $(3)$, with $Q=\left[\begin{array}{ll}1 & 0 \\ 0 & 1\end{array}\right], R=0.01$, $N_{u}=N_{u}=N_{c}=2, y_{\min }=-\infty, y_{\max }=+\infty . P$ solves the Lyapunov equation $P=A^{\prime} P A+Q$. The MPC controller is globally asymptotically stabilizing. In fact, it is easy to show that the value function is a Lyapunov function of the system. The closed loop response from the initial condition $x(0)=\left[\begin{array}{ll}1 & 1\end{array}\right]^{\prime}$ is shown in Fig. 1.

The Mp-QP problem associated with the MPC law has the form (5) with

$$
\begin{aligned}
& H=\left[\begin{array}{cc}
0.0196 & 0.0063 \\
0.0063 & 0.0199
\end{array}\right], F=\left[\begin{array}{cc}
0.1259 & 0.0679 \\
0.0922 & -0.0924
\end{array}\right] \\
& G=\left[\begin{array}{cc}
1 & 0 \\
-1 & 0 \\
0 & 1 \\
0 & -1
\end{array}\right], W=\left[\begin{array}{l}
2 \\
2 \\
2 \\
2
\end{array}\right], E=\left[\begin{array}{ll}
0 & 0 \\
0 & 0 \\
0 & 0 \\
0 & 0
\end{array}\right]
\end{aligned}
$$

The solution was computed by our mp-QP solver in $3.46 \mathrm{~s}$ on a Pentium II-300 running Matlab 5.3, and the corresponding polyhedral partition of the state-space is depicted in Fig. 1. The MPC law is 

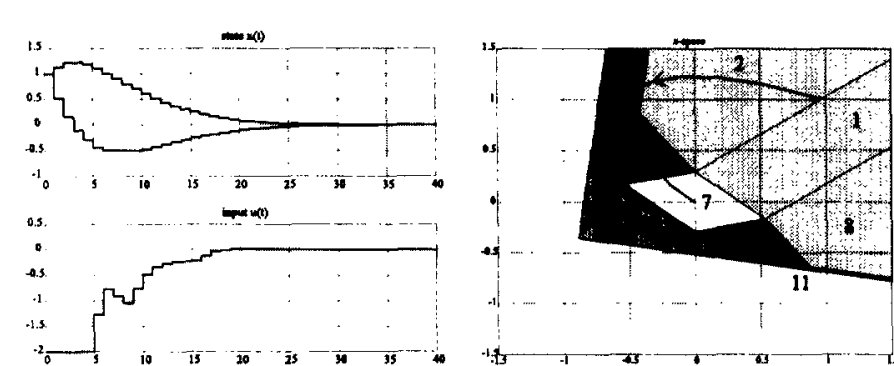

Figure 2: Additional constraint $x_{t+k \mid t} \geq-0.5$. Closedloop MPC and partition of the state-space

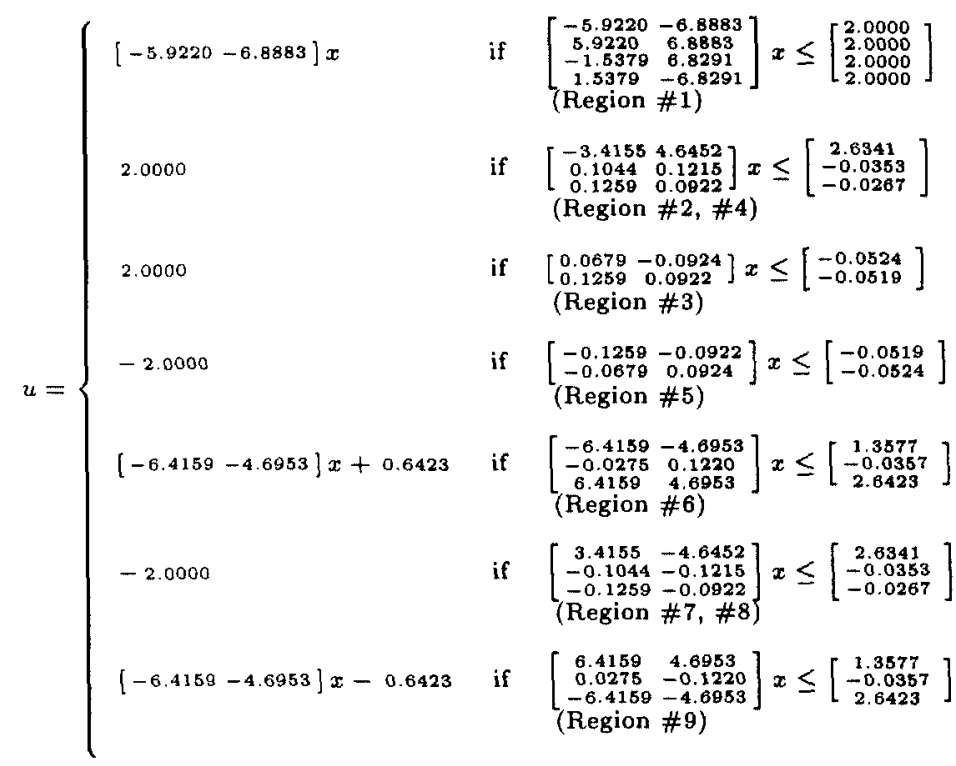

Region \#1 corresponds to the unconstrained linear controller, regions \#2, \#3, \#4 and \#5, \#7, \#8 correspond to the saturated controller, and regions \#6 and \#9 are transition regions between the unconstrained and the saturated controller. Note that the mp-QP solver provides three different regions $\# 2$, \#3, \#4, although in all of them $u=u_{t}^{*}=2$. The reason for this is that the second component of the optimal solution, $u_{t+1}^{*}$, is different, in that $u_{t+1}^{*}=[-3.41554 .6452] x(t)-0.6341$ in region $\# 2, u_{t+1}^{*}=2$ in region $\# 3$, and $u_{t+1}^{*}=-2$ in region \#4. Moreover, note that regions \#2 and \#4 are joined, as their union is a convex set, but the same cannot be done with region \#3, as their union would not be a convex set, and therefore cannot be expressed as one set of linear inequalities.

The same example is repeated with the additional constraint on the state $x_{t+k \mid t} \geq x_{\min }, x_{\min } \triangleq\left[\begin{array}{l}-0.5 \\ -0.5\end{array}\right]$, $k=1$. The closed-loop behavior from the initial condition $x(0)=\left[\begin{array}{ll}1 & 1\end{array}\right]^{\prime}$ is depicted in Fig. 2. The MPC controller was computed in $10.33 \mathrm{~s}$. The polyhedral partition of the state-space corresponding to the modified MPC controller is depicted in Fig. 2. The partition consists now of 11 regions. Note that there are feasible states smaller than $x_{\min }$, and vice versa, infeasible states $x \geq x_{\min }$. This is not surprising. For instance, the initial state $x(0)=[-0.6,0]^{\prime}$ is feasible for the MPC controller (which checks state constraints at time $t+k, k=1$ ), because there exists a feasible input such that $x(1)$ is within the limits. On the contrary, for $x(0)=[-0.47,-0.47]$ no feasible input is able to produce a feasible $x(1)$.

\section{Acknowledgments}

This research was supported by the Swiss National Science Foundation. We wish to thank Nikolaos A. Bozinis for his help with the implementation of the algorithm.

\section{References}

[1] M.S. Bazaraa, H.D. Sherali, and C.M. Shetty. Nonlinear Programming - Theory and Algorithms. John Wiley \& Sons, Inc., New York, second edition, 1993.

[2] A. Bemporad. Reducing conservativeness in predictive control of constrained systems with disturbances. In Proc. 37th IEEE Conf. on Decision and Control, pages 1384-1391, Tampa, FL, 1998.

[3] A. Bemporad, F. Borrelli, and M. Morari. Piecewise linear optimal controllers for hybrid systems. In Proc. American Contr. Conf., 2000.

[4] A. Bemporad, K. Fukuda, and F.D. Torrisi. On convexity recognition of the union of polyhedra. In Proc. Int. Conf. on Advances in Convex Analysis and Global Optimization, Samos, Greece, 2000.

[5] A. Bemporad, M. Morari, V. Dua, and E. N. Pistikopoulos. The explicit linear quadratic regulator for constrained systems. Technical Report AUT99-16, Automatic Control Lab, ETH Zürich, Switzerland, 1999.

[6] F. Borrelli, A. Bemporad, and M. Morari. A geometric algorithm for multi-parametric linear programming. Technical Report AUT00-06, Automatic Control Laboratory, ETH Zurich, Switzerland, February 2000.

[7] H.W. Chiou and E. Zafiriou. Frequency domain design of robustly stable constrained model predictive controllers. In Proc. American Contr. Conf., volume 3, pages 2852-2856, 1994.

[8] V. Dua and E. N. Pistikopoulos. An algorithm for the solution of multiparametric mixed integer linear programming problems. Annals of Operations Research, to appear.

[9] A V. Fiacco. Introduction to sensitivity and stability analysis in nonlinear programming. Academic Press, London, U.K., 1983.

[10] T. Gal. Postoptimal Analyses, Parametric Programming, and Related Topics. de Gruyter, Berlin, 2nd ed. edition, 1995.

[11] E.G. Gilbert and K. Tin Tan. Linear systems with state and control constraints: the theory and applications of maximal output admissible sets. IEEE Trans. Automatic Control, 36(9):1008-1020, 1991.

[12] S.S. Keerthi and E.G. Gilbert. Optimal infinite-horizon feedback control laws for a general class of constrained discretetime systems: stability and moving-horizon approximations. $J$. Opt. Theory and Applications, 57:265-293, 1988.

[13] M.V. Kothare, P.J. Campo, M. Morari, and C.N. Nett. A unified framework for the study of anti-windup designs. Automatica, 30(12):1869-1883, 1994.

[14] J.B. Rawlings and K.R. Muske. The stability of constrained receding-horizon control. IEEE Trans. Automatic Control, 38:1512-1516, 1993.

[15] M. Sznaier and M.J. Damborg. Suboptimal control of linear systems with state and control inequality constraints. In Proc. 26th IEEE Conf. on Decision and Control, volume 1, pages $761-762,1987$.

[16] K.T. Tan. Maximal output admisible sets and the nonlinear control of linear discrete-time systems with state and control constraints. PhD thesis, University of Michigan, 1991.

[17] E. Zafiriou. Robust model predictive control of processes with hard constraints. Computers \& Chemical Engineering, 14(4/5):359-371, 1990. 